1 Keywords: Type 2 diabetes, community pharmacy, adherence, drop-in clinic,

2 medicine use review, MUR, United Kingdom

3

$4 \quad$ Impact of findings on practice

5 - Medical practice staff were effective at signposting poorly controlled patients to 6 the drop-in clinic.

7 - Increased collaboration with the medical practice and the presence of a second

8 pharmacist to support dispensary activities may be necessary to facilitate this $9 \quad$ kind of service.

10 - Pharmacists made a number of recommendations to participants and medical 11 practices and the clinic was highly regarded by both participants and 12 pharmacists.

13 
An estimated 17.5 million people were diagnosed with a long term condition in the UK in 2005 [1]. In 2009, 2.1 million were diagnosed with type 2 diabetes and this figure is predicted to rise to 3.2 million (5.9\% prevalence) by 2020 [2]. The majority of patients (85\%) are diagnosed with type 2 diabetes which is largely controlled by oral medication [3].

The main clinical marker for type 2 diabetes is $\mathrm{HbA} 1_{\mathrm{c}}$ which, according to national guidelines should be maintained below 7.5\% [3]. However, recent UK National Health Service (NHS) figures suggest that approximately only $65 \%$ of patients are achieving this target, along with only $40 \%$ of patients achieving their target blood pressure and $42 \%$ their target cholesterol [4]. In the UK, the National Institute for Health and Care Excellence (NICE), has published guidelines for the management of patients with type 2 diabetes, which includes prescribing guidelines for blood pressure and cholesterol treatment [3]. An audit of prescribing for type 2 diabetes has demonstrated that it is generally in accordance NICE guidance [5]. Therefore, the failure of patients to achieve therapeutic targets may be related to medicine doses not being optimised or sub-optimal medication adherence as well as diet and lifestyle problems.

In 2008 , an average adult visited a community pharmacy 16 times per year with $86 \%$ of the population visiting at least once per year, $78 \%$ of those for health-related reasons [6], thereby providing an opportunity for monitoring and intervention. With the emphasis on both the prescribing of correct medicines and the need for patients to adhere to their medicines, it seems appropriate that a pharmacist can contribute to the care of patients with type 2 diabetes. The majority of patients with type 2 diabetes will be treated with medication dispensed from a community pharmacy in primary 41 care. 
43 In several UK Government policy documents, community pharmacy is specifically

44 targeted as a profession that can be used to improve the care of patients with long

45 term conditions [1, 6, 7]. Pharmacists are already engaged in providing Government-

46 funded, non-appointment based adherence interventions such as the medicine use

47 reviews (MUR) to patients with chronic conditions. The MUR is a consultation

48 generally lasting up to 20 minutes during which the patient is given the opportunity to

49 discuss their thoughts and experiences of their prescribed medicines. If any

50 problems are identified, the pharmacist then agrees a strategy for resolution which

51 may include referral back to the patient's physician. This review does not require

52 access to the patient's medical notes. These services are not condition specific and

53 have a relatively limited evidence base. It may, therefore, be more appropriate to

54 target a specific condition and provide a more tailored service than those currently

55 offered.

International studies have highlighted that the community pharmacist may have a role in addressing adherence and other concerns in this population of patients leading to improved blood glucose control [8-15]. A UK study has indicated a significant difference in $\mathrm{HbA} 1_{\mathrm{c}}$ between the intervention and control groups using RCT methodology in two pharmacies [16]. The intervention, aimed at poorly controlled patients with type 2 diabetes, involved regular monitoring and consultations with the community pharmacist for 12 months for a total of six consultations. The study was mainly conducted in one pharmacy with two permanent pharmacists and a part time nurse. It consisted of a targeted medicine use review to discuss any adherence problems if appropriate, lifestyle modification and referral to the GP if necessary. It also included education about diabetes and its complications. This was an intensive, repeated intervention service with significant time input and no cost effectiveness analysis [16]. We believe, to make this service widely available to patients there would need to be significant change to the ways pharmacists are

71 currently remunerated. This is because UK pharmacy remuneration is dominated by 72 fees for dispensing medicines thus spending long periods of time with patients 
means fewer prescriptions can be checked or dispensed. An alternative funding approach would be to take the existing MUR framework (which only requires a oneoff, 20 minute consultation) and make it more specific to patients with diabetes. This will be a small step change in the provision of pharmacy services that would not require a significant operational restructure.

In a series of focus groups with patients with type 2 diabetes, participants drawing on their experience of living with diabetes indicated that they would be willing to engage with a community pharmacy service aimed at improving their condition providing that the pharmacist was working in co-operation with the medical practice and they were not violating the natural line of treatment between them and the doctor [17].

All participants in these focus groups identified that they wanted to take responsibility for their own condition but occasionally they had needs for information and wanted to be able speak to a suitably qualified healthcare professional. As such, participants liked the ease and convenience of speaking to the pharmacist and the lack of need for appointment bookings.

This preparatory work led to the design of a diabetes drop-in clinic in the community pharmacy setting that involved identification of poorly controlled patients by the medical practice, no appointment system and a focus on adherence and lifestyle advice. This paper provides details on the results of a feasibility study.

\section{Aims of the study}

To determine whether a community pharmacy diabetes drop-in clinic is feasible and acceptable to patients with poorly controlled type 2 diabetes and assessing which outcome measures would be appropriate for a larger study and describing the content of the consultations. 


\section{Ethical Approval}

103 Essex NHS Research Ethics Committee (Ref: 11/EE/0494) and NHS Norfolk

104 Research and Development committee (Ref: 2011IC01) approved this study.

105

106

\section{Method}

107 As this was a feasibility study, community pharmacies and medical practices were

108 selected based on convenience and contacts that existed between pharmacists and 109 the academic institution.

111 The diabetes pharmacy drop in clinic specifically targeted patients who were poorly 112 controlled with respect to one or more of $\mathrm{HbA}_{1 \mathrm{c}}(>59 \mathrm{mmol} / \mathrm{mol})$, blood pressure $113(>140 / 80 \mathrm{mmHg})$ or lipids ( $>5 \mathrm{mmol} / \mathrm{L})$ as defined by a national incentive scheme.

114 Medical practice staff identified eligible patients on behalf of the research team. The 115 staff were provided with pre-filled envelopes enclosing a letter from the practice 116 partners and a leaflet advertising the service and were asked to mail these to all 117 identified poorly controlled patients. The leaflet contained information including clinic 118 times and what was involved when patients attended the pharmacy. The researcher 119 had no access to medical records for this process. Informed patient consent was 120 obtained when the patient presented in the pharmacy and this allowed the 121 pharmacist to collect biometric data from the medical practice for the purposes of the 122 research.

124 The clinic was conducted in five pharmacies with the regular pharmacist in the 125 private consultation room located in the pharmacy. Two pharmacies used a 126 consultation room in the adjoining medical practice as they did not have one on the 127 pharmacy premises. This was usual practice for those pharmacists when conducting 128 MURs. Pharmacists were given extra training in order to provide the service which 
154 The consultation was then conducted by the community pharmacist in the 155 consultation room for a duration determined by the patient. The pharmacist was 156

included a self-directed learning package and a short face-to-face session with the lead researcher. The clinic was conducted for a four-hour period once a week for four weeks (six weeks in one pharmacy) and patients were able to attend without making a prior appointment. The clinic times were selected to ensure that a variety of days of the week (including Saturdays) and times (morning and afternoon) were covered. The aim was to recruit 30-40 participants between the five pharmacies. A second pharmacist (MT) provided dispensary support to the intervention pharmacist. Patients also had the opportunity to visit the pharmacy outside of the clinic times but were informed that they may have to wait a short while to see the pharmacist.

Before undertaking the consultation participants were asked to complete a short questionnaire containing three validated questionnaires: the Beliefs about Medicines Questionnaire (BMQ) [18], the Satisfaction with Information about Medicines questionnaire (SIMS) [19] and Morisky measure of adherence (MMAS-4) [20] combined with questions regarding how many times and why they have used the community pharmacy over the preceding three months. The MMAS-4 is composed of four questions surrounding a patient's medicine taking behaviours. A score of 4 on this scale is interpreted as the patient being fully adherent while less than four indicates partial adherence. This information was then used by the pharmacist during the consultation with the participant. These outcome measures were selected as the service was designed based on existing community pharmacy services which largely involve information provision and adherence advice. There is evidence to suggest that information satisfaction is related to adherence [19] and that this can also be related to a patient's beliefs and concerns surrounding a particular medicine [21]. asked to document the content of the consultation on a standard form. As a feasibility 
157

158 the research team but led by the patient from their discussion with the pharmacist

159 and their responses to the baseline questionnaire.

160

161 Post consultation, the participants were asked to complete a satisfaction

162

163

164

177 The pharmacist interviews were transcribed and coded by the researcher and

178 themes were developed using content analysis as described in the literature [22]. A

179 second researcher also read the transcripts and familiarised themselves with the

180 participants responses. The two researchers had discussions surrounding the

181 themes to arrive at a consensus and resolve any conflicting views. 


\section{Results}

184 Five pharmacies (three independents and two chain pharmacies) and three medical 185 practices were recruited in three locations across Norfolk, UK. Two of the three 186 independent pharmacies were owned by the medical practice that also participated in

187 the project. None of the medical practices had an established relationship with the 188 academic institution at the outset of the study.

189

190 The medical practices identified and posted the invitation letter and information sheet

191 to 342 potential participants. Thirty-three participants (9.6\% response rate) were

192 recruited in four of the five pharmacies with each pharmacy seeing between zero and 193 five participants during each four-hour session. The demographics of the recruited

194 participants are detailed in table 1. The mean (SD) time for the consultation was 32.5

195 (12.0) minutes but ranged from 15 minutes to 65 minutes. As part of the

196 consultations, pharmacists discussed a wide variety of topics and made a number of

197 referrals to the medical practice including:

198

- Providing information sheets on diet and lifestyle

- Advising participants on portion size

- Information provision on medication

- Identification and reporting of adherence issues

- Changes to formulation to aid adherence

- Requests for alternative or additional medicines for cholesterol and other conditions

208 Insert table 1. 
210 All participants completed the baseline questionnaire and the team received 26

211 (79\%) follow-up questionnaires. There was no difference in any of the questionnaire

212 measures between baseline and follow-up (tables 2 and 3) apart from the types of

213 topics that participants were prepared to talk to the pharmacist about. The number of

214 patients classed as adherent rose from $61.5 \%$ at baseline to $76.9 \%$ at follow-up.

215

216 Insert tables 2 and 3.

217

218 Satisfaction questionnaire

219 In total, 27 completed questionnaires were returned. These results demonstrate that 220 participants were extremely satisfied with the service that they received and they

221 most would recommend the service to another patient with type 2 diabetes. In

222 response to the question regarding how useful the service was to helping manage

223 their diabetes, $100 \%$ agreed that it was some or a lot of help. Nearly $60 \%$ of

224 participants stated that this experience would make them more likely to consult their 225 pharmacist in future about other conditions with nearly $90 \%$ stating that the length of 226 the consultation was about right. A summary of the other questions asked can be 227 found in figure 1 and demonstrates that all aspects of the service and study process 228 were well received.

229

230 Insert figure 1.

231

232 Pharmacist de-brief interviews

233 The pharmacist debrief interviews centred on three areas of discussion: training

234 provision, conduct of the service and the benefits arising from the service.

235

236 Training provision 
237 The participating pharmacists identified that the training provision for the service

238 which consisted of a self-directed learning package and a short face-to-face training 239 session was adequate to cover their needs for the study. As part of the face-to-face 240 element, pharmacists were informed of the previous work from the focus groups with 241 patients. This helped them to contextualise the clinic within their practice and tailor 242 their consultations with this information in mind. One pharmacist identified that, in her 243 opinion, interaction with her peers would have been useful to determine how each of 244 them was going to implement the service and what they had learnt as a result of the 245 training in diabetes and study documentation thus far whereas another pharmacist 246 wanted interaction with other healthcare professionals. "I personally would've liked... time with either the diabetes nurse or one of the doctors at the practice er just to clarify er sort of their guidelines and what they were trying to achieve with their patients." Pharmacist 3

There was a need for this pharmacist to integrate further with the medical practice and determine their patterns and guidelines for treatment as he did not want to go against the wishes of the practice nurse or GP when making suggestions to them for treatment alterations.

Conduct of the service

258 Once in the consultation, pharmacists identified a number of topics that patients 259 wanted to cover and these varied for each pharmacist.

"Quite a few people wanted to know about like the prognosis of diabetes...they didn't quite realise that they would be on medication for like a long time" Pharmacist 2 
265 This pharmacist appeared surprised at the content of the consultation, expecting

266 participants to focus on medicines but instead wanting to discuss other matters

267 surrounding their condition. With the pharmacist below, their perception was that

268 participants just wanted reassurance that they were doing the right things to control

269 their diabetes.

270 required for the study.

"I think most people came with some ideas, some had things they just wanted reassurances about other people just came to say their diabetes is fine and explain their medications..." Pharmacist 5

The pharmacists felt that because of this wide variation in topics covered during the consultation, this meant they were sat with the patient for an extended period of time, which they felt had its benefits but could only be achieved because another pharmacist was covering their dispensary workload.

“They don't normally get to spend a long time talking to the doctor or nurse, they are often rushed... I think it's quite well received by patients...I think it would be very difficult to run that kind of service if I didn't have any locum cover or second pharmacist cover... they [patients] feel less intimidated disturbing what you are doing." Pharmacist 5

291 Benefits arising out of the study 
292 Pharmacists identified that participating in this study had benefits to patients,

293 themselves as healthcare professionals and their interaction with the medical

294 practice. Pharmacists highlighted that a positive aspect of the study was that they

295 had had participants return to them after the consultation to update them on their

296 progress, which is something that, as pharmacists, they are not used to.

“...we've already had somebody come in this morning to say how his levels... have improved as a result of just having a chat. I think it is fantastic if we... get away from checking prescriptions and providing a service like this its

Most pharmacists highlighted that participating in the study was beneficial to their wider practice as well as the drop-in clinic and that it had given them more confidence to speak to this group of patients. One final benefit that was highlighted was the increased collaboration with the medical practice. great" Pharmacist 4

313 All of the pharmacists saw this kind of service as benefitting the relationship with the 314 medical practice and demonstrating where the community pharmacist could help 315 when trying to control patients with type 2 diabetes. They also stated that this would 316 help to raise the profile of pharmacy more generally within the medical practice, 317 which could only be positive for pharmacy. 


\section{Discussion}

320 The primary aim of this study was to determine if a drop in clinic based on patient 321 preferences aimed at those with poorly controlled type 2 diabetes and conducted in 322 the community pharmacy was feasible and acceptable to patients and pharmacists. It 323 has demonstrated that patients will access community pharmacy services if identified 324 via the medical practice and that they have significant information needs in relation to 325 their condition and medicines that can be addressed by the pharmacist. It also set 326 out to examine which outcome measures may be appropriate in a future study and 327 ascertain the focus of the consultation.

In terms of feasibility testing, this study has been successful. The medical practices were willing to approach patients on behalf of the service, pharmacists could conduct the consultations and patients found them acceptable and were willing to engage

332 with the process. However, this study could only be conducted with a second 333 pharmacist that allowed the intervention pharmacist to spend the length of time they 334 did with the participants and is unclear how cost-effective this would be on a larger scale. It would also need to be investigated whether this length of time spent with the patient represents a good use of resources. On reflection, the one pharmacy that did not recruit any patients felt that this was due to the lack of prescription volume from the medical practice associated with the study.

340 Questionnaire results from baseline to follow-up demonstrated no differences in the measures of satisfaction with information or beliefs about medicines. There was a slight increase in the percentage of participants classed as adherent at the end of the study. This indicates that these measures may not be appropriate for a larger study.

345 In a larger study, the primary outcome measure would be $\mathrm{HbA}_{1 \mathrm{C}}$ and this would need 346 to be collected for some time after the end of the study and any changes made 
requested by the pharmacist as a result of the clinic would need to be followed-up to

348 determine their implementation rate. The extent to which both of these can be

349 achieved was not tested during this study. Adherence was characterised using a self-

350 report method which may be less reliable than other forms of adherence

351 measurement e.g. prescription refill data [23]. Both $\mathrm{HbA}_{1 \mathrm{C}}$ and refill data may be

352 more useful outcome measures for a future study and would also reduce the

353 participant questionnaire burden. Along with self-report adherence, participants self-

354 selected for this service and they were therefore more likely to be motivated to

355 engage with an intervention aimed at their condition. However, despite this limitation

356 the study only invited patients who were poorly controlled and therefore any

357 improvement in their condition will be beneficial to the patient and the NHS.

359 Participant satisfaction with the service was high with most suggesting that they

360 thought it would help them manage their diabetes better and that this kind of service

361 should be available to all patients. They identified that the pharmacists appeared

362 knowledgeable, professional and approachable with some participants noting that the

363 pharmacist did not appear to rush them and was not distracted by other work in the

364 dispensary. This has previously been identified as a problem and may indicate a

365 potential reason why a patient may not engage with the pharmacist [17]. One

366 limitation of the questionnaire includes the phrasing of the questions regarding

367 community pharmacy use in the last three months. Participants may have included

368 the study consultation in their responses and therefore these results should be

369 interpreted with caution.

371 From the de-brief interviews, pharmacists stated that they enjoyed providing the

372 drop-in clinic as it allowed them to use the knowledge that they had learnt from their

373 training. This allowed them to interact with patients for longer and they especially

374 enjoyed the feedback and hearing from patients about their progress once the study 
had finished. In terms of the feedback on the consultation, the pharmacists identified

376 that one of the most important aspects to the service was the dispensary support

377 provided as part of the research. This enabled them to focus on the patient and not

378 feel distracted by events in the dispensary. Another central point that the pharmacists

379 focused on was the need to talk to the practice nurse or doctor about local treatment

380 guidelines to ensure that they were not providing conflicting advice. This

381 demonstrates the need for pharmacists to be better integrated within the primary

382 healthcare team and is something the patients have identified as important [20].

384 The study achieved a response rate of $9.6 \%$ from the postal invitation, something 385 that has implications for generalisability of the results. A low response rate such as 386 this may imply that only motivated patients were encouraged to participate in the 387 study and therefore these patients may not be representative of the wider population 388 with diabetes. This response rate for also has implications for calculating the required 389 number of participants for a larger study.

391 The consultations themselves lasted significantly longer than GP consultations [24] 392 with the mean time at approximately 32 minutes. This allowed the pharmacist to 393 spend longer with the participants discussing all aspects of their care but may prove 394 an expensive intervention when compared to a similar nurse-led service [25], 395 however the pharmacist is more likely to have capacity for this type of intervention. 396 This could have implications for this type of community pharmacy service in primary 397 care and means that there will need to be a further defining of the intervention in 398 order to make it distinct from current nurse provision, which is less expensive. If 399 pharmacists have the time to spend with patients in this manner, then it may be 400 appropriate for the intervention to focus on using behaviour change techniques, 401 which have been found to have a positive effect on adherence [26] rather than on a 402 wider variety of (unfocussed) topics. 
404 However, there still remain unanswered questions regarding the community

405 pharmacist's role in this group of patients, particularly with reference to the role of the

406 practice or diabetes nurse in the UK. As a result of this work and the work of others

407 [16], it appears sensible to undertake further exploratory work to determine the type

408 of intervention pharmacists should be providing to this group of patients.

409

\section{Conclusion}

411 The diabetes community pharmacy drop-in clinic was well received by patients and

412 pharmacists and was feasible to conduct in this particular setting. However, there still

413 remain questions regarding the input of the community pharmacist in the care of this

414 group of patients, particularly with such a strong nurse-led service already provided

415 to them in the UK setting. With a significant number of patients still remaining

416 uncontrolled with respect to national guidelines it is therefore appropriate to conduct

417 further work to determine if and how the pharmacist can support the wider primary

418 care team in improving treatment for patients with type 2 diabetes.

Acknowledgements The research team would like to thank the medical practices

421 and pharmacies for volunteering to participate in this project. The pharmacies and

422 medical practices were not paid for participating in the study.

424 Funding: This work was funded jointly by Numark Pharmacy Ltd and the UEA.

426 Conflicts of interest: none

\section{References}

430 1. Department of Health, Choosing health through pharmacy - a programme for pharmaceutical public health 2005-2015. 2005, The Stationery Office: London. 
2. The Health and Social Care Information Centre \& The Yorkshire and Humber Public Health Observatory, Prescribing for diabetes in England - An update: 2002-2008. 2009.

3. NICE, Type 2 diabetes: The management of type 2 diabetes (CG87). 2009, National Institute of Health and Clinical Excellence,: London.

4. Health \& Social Care Information Centre, National Diabetes Audit 2011-2012 Report 1: Care Processes and Treatment Targets. 2013, Health \& Social Care Information Centre, : London.

5. Twigg, M.J., et al., An audit of prescribing for type 2 diabetes in primary care: optimising the role of the community pharmacist in the primary healthcare team. Primary Health Care Research \& Development, 2013. 14(03): p. 315319.

6. Department of Health, Pharmacy in England: building on strengths delivering the future. 2008, The Stationery Office: London.

7. Department of Health, $A$ vision for pharmacy in the new NHS. 2003, The Stationery Office: London.

8. Correr, C.J., et al., Effects of a pharmacotherapy follow-up in community pharmacies on type 2 diabetes patients in Brazil. International Journal of Clinical Pharmacy, 2011. 33(2): p. 273-280.

9. Fornos, J., et al., A pharmacotherapy follow-up program in patients with type2 diabetes in community pharmacies in Spain. Pharmacy World and Science, 2006. 28(2): p. 65-72.

10. Krass, I., et al., The pharmacy diabetes care program: Assessment of a community pharmacy diabetes service model in Australia. Diabet Med, 2007. 24(6): p. 677-683.

11. Krass, I., et al., Diabetes Medication Assistance Service Stage 1: impact and sustainability of glycaemic and lipids control in patients with Type 2 diabetes. Diabet Med, 2011. 28(8): p. 987-993.

12. Mehuys, E., et al., Effectiveness of a community pharmacist intervention in diabetes care: a randomized controlled trial. J Clin Pharm Therapeut, 2011. 36(5): p. 602-613.

13. Oyetayo, O., et al., The Hispanic Diabetes Management Program: Impact of community pharmacists on clinical outcomes. JAPhA, 2011. 51(5): p. 623-6.

14. Taylor, S.J., et al., A cost-effectiveness analysis of a community pharmacistinitiated disease state management service for type 2 diabetes mellitus. Int $\mathrm{J}$ Pharm Pract, 2005. 13: p. 33-44.

15. Wermeille, J., et al., Pharmaceutical care model for patients with type 2 diabetes: integration of the community pharmaicst into the diabetes team - a pilot study. Pharm. World Sci., 2004. 26: p. 18-25.

16. Ali, M., et al., Impact of community pharmacy diabetes monitoring and education programme on diabetes management: a randomized controlled study. Diabetic Med., 2012: p. e326 - e333.

17. Twigg, M., et al., The current and future roles of community pharmacists: Views and experiences of patients with type 2 diabetes. Res Soc Admin Pharm, 2012. 9(6): p. 777-789.

18. Horne, R., J. Weinman, and M. Hankins, The beliefs about medicines questionnaire: The development and evaluation of a new method for assessing the cognitive representation of medication. Psychol Health, 1999. 14(1): p. 1-24.

19. Horne, R., M. Hankins, and R. Jenkins, The Satisfaction with Information about Medicines Scale (SIMS): a new measurement tool for audit and research. Quality in Health Care, 2001. 10(3): p. 135-140.

20. Morisky, D., L. Green, and D. Levine, Concurrent and predictive validity of a self-reported measure of medication adherence. Med Care, 1986. 24(1): $p$. 67-74. 
21. Horne, R. and J. Weinman, Patients' beliefs about prescribed medicines and their role in adherence to treatment in chronic physical illness. J Psychosomat Res, 1999. 47(6): p. 555-567.

22. Graneheim, U. and B. Lundman, Qualitative content analysis in nursing research: concepts, procedures and measures to achieve trustworthiness. Nurse Educ Today, 2004. 24(2): p. 105.

23. Matsuyama, J.R., B.J. Mason, and S.G. Jue, Pharmacists' interventions using an electronic medication-event monitoring device's adherence data versus pill counts. Ann Pharmacother, 1993. 27(7-8): p. 851-855.

24. Carr-Hill, R., et al., Do minutes count? Consultation lengths in general practice. J Health Serv Res Policy, 1998. 3(4): p. 207-213.

25. Curtis, L., Unit Costs of Health and Social Care 2012, Personal Social Services Research Unit and University of Kent, Editors. 2012.

26. Easthall, C., F. Song, and D. Bhattacharya, A meta-analysis of cognitivebased behaviour change techniques as interventions to improve medication adherence. BMJ Open, 2013. 3(8). 
Do you think the pharmacist is the right person to conduct this type of clinic?

Do you think the pharmacy was the right place for this type of clinic?

In terms of content, do you think the first questionnaire asked the right questions for you?

Did you think that a direct leaflet was the best way to inform you of the clinic? $\mathrm{N}=25$

Would you recommend this service to other patients with type 2 diabetes?

Would you use this service again?

Do you think this service, or a service like it, could be useful for other patients with diabetes or other conditions?

Did you feel the information given was at a level that you could understand?

Were you satisfied with the information you received during the consultation?

Did you find it useful to speak to the pharmacist about your condition?

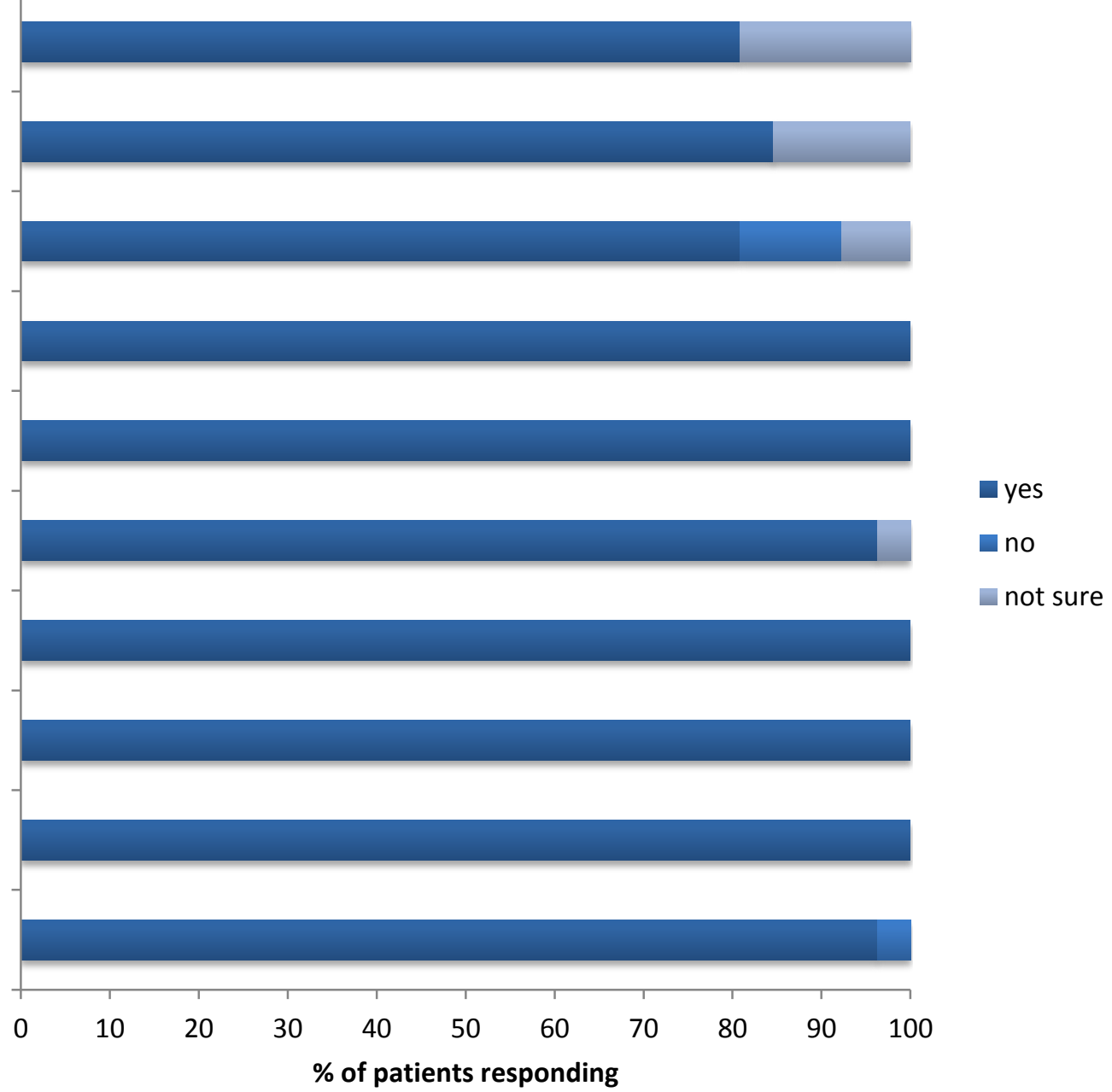

Figure 1 Responses that required a yes/no/not sure answer from the feedback questionnaire 
Table 1 Participant Demographics

\begin{tabular}{|lccc|}
\hline & N & Mean (SD) & $\begin{array}{c}\text { \% of patients } \\
\text { uncontrolled }\end{array}$ \\
\hline Distance travelled to clinic (miles) & 33 & $3.1(2.7)$ & $\mathrm{n} / \mathrm{a}$ \\
Most recent HbA 1 c result (mmol/mol) & $27^{*}$ & $63.5(13.2)$ & 44.5 \\
Most recent SBP result $(\mathrm{mmHg})$ & $27^{*}$ & $133.6(21.7)$ & 74.1 \\
Most recent DBP result $(\mathrm{mmHg})$ & $27^{*}$ & $78.8(16.1)$ & 81.5 \\
Most recent total cholesterol result (mmol/L) & $27^{*}$ & $4.4(1.4)$ & 48.1 \\
Number of medicines prescribed & $29^{*}$ & $8.8(4.2)$ & $\mathrm{n} / \mathrm{a}$ \\
Years since diagnosis & $29^{*}$ & $8.1(5.0)$ & $\mathrm{n} / \mathrm{a}$ \\
\hline
\end{tabular}

${ }^{*}$ Data unobtainable for some participants presenting at pharmacy 4. SBP: systolic blood pressure; DBP: diastolic blood pressure; data normally distributed. 
Table 2 Baseline and follow-up questionnaire data

\begin{tabular}{|lcc|}
\hline Measure & $\begin{array}{c}\text { Before } \mathrm{N}=33 \\
\text { (median (quartiles))* }\end{array}$ & $\begin{array}{c}\text { After } \mathrm{N}=26 \\
(\text { median (quartiles))* }\end{array}$ \\
\hline $\mathrm{BMQ}$ - necessity scale /25 & $20(17-23)$ & $20(17-22)$ \\
$\mathrm{BMQ}-$ concerns scale /25 & $14(11-18)$ & $16(12.75-18)$ \\
$\mathrm{BMQ}-$ differential score & $6(2-9)$ & $4.5(-0.25-8.25)$ \\
SIMS - actions and usage & $7(4-8)$ & $7(5-9)$ \\
score /9 & $4(2.5-7)$ & $5.5(2.25-7.25)$ \\
SIMS - potential problems & $11(8-13)$ & $11(7.75-16)$ \\
score /8 & & \\
SIMS - total score /17 & &
\end{tabular}

BMQ: beliefs about medicines; SIMS: satisfaction with information about medicines scale 
Table 3 Community pharmacy use before and after the study

\begin{tabular}{|l|cc|}
\hline $\begin{array}{l}\text { Not counting today, how many } \\
\text { times in the last three months }\end{array}$ & $\begin{array}{c}\text { Before } \mathrm{n}=33 \\
\text { Mean (SD) }\end{array}$ & $\begin{array}{c}\text { After } \mathrm{n}=26 \\
\text { Mean (SD) }\end{array}$ \\
\cline { 2 - 3 } $\begin{array}{c}\text { hou: } \\
\text { Visited the pharmacy }\end{array}$ & $3.37(2.82)$ & $3.37(2.65)$ \\
Spoken to the pharmacist & $0.96(1.54)$ & $1.91(2.51)$ \\
\hline What have you spoken to the & \% responding 'yes' $\%$ responding 'yes' \\
pharmacist about? & 0 & 38.5 \\
Your condition & 15.2 & 34.6 \\
Your medication & 18.2 & 23.1 \\
Over-the-counter advice & 6.1 & 19.2 \\
Lifestyle & 9.1 & 26.9 \\
Dietary advice & 12.1 & 26.9 \\
Other medical conditions & 9.1 & 19.2 \\
Minor ailments & 21.2 & 53.8 \\
\hline Medicine supply & & \\
\hline
\end{tabular}

